\title{
Time to cut regulations that protect only regulators
}

Researchers and their live subjects in US universities are valuably protected by some regulatory processes but pointlessly undermined by others. It is time to streamline or scrap the latter.

$\mathrm{T}$ he tragic deaths of two young people in clinical trials at the University of Pennsylvania and Johns Hopkins University have had a chilling impact on the scientific community, by highlighting what can happen when well-intentioned research goes wrong. Attention in the United States has correspondingly been focused on the workings of the Institutional Review Boards (IRBs) that review and approve human experimentation.

No doubt all this will mean even more federally mandated rules and regulations that US scientists and institutions will have to follow in order to conduct scientific research legally. Unfortunately, what seems to happen whenever such controversies arise is a blanket application of 'the rules', with no leeway for common sense. The overall effect is that researchers and institutions alike are drowning in paperwork. Sadly, much of the documentation bears little relation to the realities of research, for it accomplishes little beyond slowing down legitimate experiments and making them harder to administer. The work still goes on - just more slowly, and at greater cost in time and money.

Such views will be discounted by some as reflecting the blinkered interests of researchers. Nature has not hesitated in the past to urge scientists to be more responsive to ethical and social concerns in this context. But it is important to discriminate between what is truly a risk to the study subject, whether human or animal, and what is not. The former category of experiment requires rigorous review, to prevent unnecessary deaths and injury. But much of the research that falls under the purview of IRBs and Institutional Animal Care and Use Committees (IACUCs) does not warrant such close scrutiny, as it causes little real risk for the study subject or, in the case of animals, involves generally accepted procedures such as immunization, taking blood or challenge with standard pathogens. The time spent by committees reviewing such routine protocols to the full extent required by law is time that could be better spent on the more controversial applications.

\section{Climate of fear}

This point has been well made in the context of IRB reviews by Robert Schooley, of the University of Colorado School of Medicine, and colleagues in various publications, but it applies to animal research as well. In one respect, the situation with IACUCs is even worse than with IRBs. Nowadays, IACUC reviews must take place before the grant application can even be peer reviewed by the National Institutes of Health (NIH). As most applications are rejected upon peer review, an enormous amount of time is wasted by IACUCs in approving animal experiments that will never be performed. The procedure used to be that an IACUC application was only necessary once a grant application had fared well enough to be approved in principle; this sensible process was changed through pressure on the NIH from animal-welfare lobbyists, leading to yet more paperwork but no real benefit to animals.

The climate under which even routine protocols are reviewed by IACUCs and IRBs is now one of fear - fear by the institution that it will be "out of compliance" with one or more aspects of the paperwork, and so subject to penalty upon audit (be that by the NIH, the Office for Human Research Protection, the US Department of Agriculture, or whatever other organization is involved). Again, the attention is on the paperwork, not on the truly important question: is the risk of the experiment to the subject worth the likely benefit of the experiment to society?

\section{Paper-trail politics}

In the legal system, precedents are important criteria for determining the outcome of cases, but this does not apply with review committees: even if a similar experiment has been approved and successfully carried out elsewhere it must be reviewed yet again in-house. This is not, in reality, to protect the animal — it is to protect the institution and, by extension, the reviewers.

There is no permitted cross-talk between committees in different institutions and no central list of approved procedures, so each institutional committee must act as an island unto itself. Indeed, if an experiment involves a collaboration between institutions, each institution (perhaps as many as three or four) must individually review the application - adding to the cost of the review but not to the welfare of the humans or animals involved. Non-controversial applications are multiply nit-picked; forms are returned to the applicant because a single word has been mistyped, or even because the wrong font has been used.

It seems as if review committees 'must' find something wrong with each and every application, so as to generate a paper trail proving the thoroughness of their review. For controversial applications this is entirely appropriate, but the 'fear factor' means that enormous effort is now expended on routine and mundane protocols. In some institutions, scientists who never work with organisms more complex than yeast and bacteria are now being forced to attend lectures on how to conduct research on humans, "just in case". This is yet another institutional response to pressure from federal agencies, but what purpose does it serve in the real world?

Does society want to support scientific research? If it does, it should find ways to make the system work efficiently as well as safely. At present, local and federal administrators and scientists alike are frustrated by what must be done to make research legal in the face of those who would have it otherwise.

There's also a price to pay for the extra workload — committee member burn-out. To use a topical analogy, how many suitcases full of underwear and socks can an airline security agent wade through before he or she is too tired to find the hidden box-cutter? It's more efficient, surely, to identify the threatening passenger before the suitcase is packed, than to pick every case apart, just to check if there's a problem. Just as travellers have to travel, scientists still have to do science. It's a sad day when the working scientist realizes that the most important person in the laboratory is not the smartest postdoc nor the hardest-working student, but the chief administrator. 